\title{
Cyclostationarity-based joint domain approach to blind recognition of SCLD and OFDM signals
}

\author{
Zhuo Sun*, Yilong Chen, Siyuan Liu and Wenbo Wang
}

\begin{abstract}
The cyclostationarity feature of modulated signals was widely applied for signal detection and parameter estimation. Previous studies on blind recognition of the modulation type of a received signal are generally performed in a single domain. This paper presents a new recognition approach through establishing a joint delay-cyclic frequency feature decision function, which exploits the different features of the second-order cyclic cumulants for orthogonal frequency division multiplexing (OFDM) signals and single carrier linear digitally modulated (SCLD) signals, in both time delay domain and cyclic frequency domain. We also propose a new statistical test method to classify the two signals under the constraint of possible error recognition, with which an optimal threshold to distinguish OFDM and SCLD signals with the highest accuracy can be theoretically derived. The simulation results are consistent with the performance analysis.
\end{abstract}

Keywords: Blind recognition; Cyclostationarity; Second-order cyclic cumulants

\section{Introduction}

Recognition of complex sonar or radar signals has been an important technology for a variety of military and commercial applications. With the new transmission schemes gradually migrating from communication systems into sonar and radar systems, such as single carrier linear digitally modulated (SCLD) and multicarrier modulation in the form of orthogonal frequency division multiplexing (OFDM), the traditional methods employing direction of arrival, carrier frequency, time arrival, pulse amplitude and width cannot meet the requirement of accurate and substantial target recognition. As a result, blind recognition of the modulation format of the signal becomes one of critical issue $[1,2]$.

Because cyclic feature embedded in the modulated signal are quite robust to both noise uncertainty and strong noise, it can differentiate various modulation types [3-5]. However, in most cyclostationary-based blind recognition of the modulation methods [6-9], they try to recognize OFDM and SCLD signals only in a single domain. However, in that situation, the difference of the second-order cyclic cumulant (CC) magnitude between OFDM and SCLD is not obvious under low signal-to-

\footnotetext{
* Correspondence: zhuosun@bupt.edu.cn

Key Laboratory of Universal Wireless Communications, Ministry of Education Beijing University of Posts \& Telecommunications, Beijing 100876, China
}

noise ratio (SNR) condition, which tremendously leads to the result that the probability of correctly recognizing the signal is not efficacious. On the other hand, different cyclostationary features between the OFDM and SCLD signals still existed in frequency domain which had not been utilized. To achieve high accuracy in recognizing the signal especially in low-SNR environments, the proposed method in this paper jointly exploits the features of signal in time and frequency domain, which was formulated as a unified feature function.

For the feature-based blind recognition methods, the decision based on the selected feature function is another one major procedure. Most decision algorithms use the asymptotically optimal test with the constant false-alarm rate (CFAR) property which is proposed in [10]. However, it only considers the probability that the noise is recognized mistakenly as OFDM signals (or SCLD signals) to setup the decision threshold, which is not optimal for the classification of OFDM and SCLD signals. This paper proposes a new statistical test method for the classification of the two kinds of signals in consideration of the total error probabilities. In the method, the quantitative relation between the probability of correct recognition and the threshold is formulated, based on which the analytical close-form expression of optimal decision threshold can then be derived. 
This paper is organized as follows: The SCLD and OFDM signal models and the analytical expressions for the second-order $\mathrm{CCs}$ and cycle frequencies (CFs) are presented in Sections 2. The proposed joint delay-cyclic frequency domain feature function-based algorithm and the corresponding statistical test are presented in Section 3. Simulation results are given and discussed in Section 4. Finally, conclusions are drawn in Section 5.

\section{Signal model and second-order cyclostationarity}

In [11], it proved that the addition of cyclic prefix (CP) to an OFDM symbol provides a useful cyclostationary feature. Meanwhile, for a SCLD signal, cyclostationarity is brought in by the pulse-shape filter.

Referring to [12], the continuous-time baseband equivalent of received SCLD signals in AWGN is expressed as follows:

$$
r_{\mathrm{SCLD}}(t)=a e^{j \theta} e^{j 2 \pi \Delta f_{c} t} \sum_{l=-\infty}^{+\infty} s_{l} g(t-l T-\varepsilon T)+\omega(t),
$$

where $a$ is the amplitude factor, $\theta$ is the carrier phase, $\Delta f_{c}$ is the frequency offset, $T$ is the symbol period, $\varepsilon(0 \leq$ $\varepsilon \leq 1)$ is the timing offset, $g(t)$ is the overall impulse response of the transmit and receive filters, $s_{l}$ is the symbol transmitted in the $l$ th symbol period, and $\omega(t)$ is a zero-mean complex Gaussian noise.

The received baseband OFDM signal is expressed as follows in [12]:

$$
\begin{aligned}
r_{O F D M}(t)= & a e^{j \theta} e^{j 2 \pi \Delta f_{c} t} \sum_{k=0}^{K-1} \sum_{l=-\infty}^{+\infty} s_{k, l} e^{j 2 \pi k \Delta f_{k}(t-l T-\varepsilon T)} g(t-l T-\varepsilon T) \\
& +w(t),
\end{aligned}
$$

where $K$ is the number of OFDM signal subcarriers, $s_{k, l}$ is the symbol transmitted on the $k$ th subcarrier over the $l$ th symbol period, $\Delta f_{K}$ is the frequency step between two adjacent subcarriers, and $T=T_{C P}+T_{u}$ is the OFDM symbol period.

The analytical closed-form expressions for the secondorder (one-conjugate) CCs and the set of CFs for SCLD signals are given respectively in [7] as follows:

$$
\begin{aligned}
& c_{r_{\mathrm{SCLD}}}(\beta ; \tau)=J(\tau) \rho^{-1} e^{-j 2 \pi \beta \varepsilon \rho} \Lambda(\tau)+c_{\omega}(\beta ; \tau) \\
& \kappa_{2,1}^{\mathrm{SCLD}}=\left\{\beta \in[-1 / 2,1 / 2) \mid \beta=l \rho^{-1}, l=1,2,3 \ldots\right\},
\end{aligned}
$$

where $\quad J(\tau)=a^{2} e^{-j \frac{2 \pi}{\rho} \Delta f_{c} T \tau} c_{s, 2,1}, \quad \Lambda(\tau)=\sum_{u=-\infty}^{+\infty} g(u) g^{*}(u+\tau)$ $e^{-j 2 \pi \beta u}, \rho$ is the sampling factor, and $c_{s, 2,1}=E\left[s_{l} l_{l+\tau / T}^{*}\right]$ represents the second-order cumulant of the signal constellation.
Similar to the SCLD signals, the second-order (oneconjugate) CCs and the set of CFs for OFDM signals are given by [13] as follows:

$$
\begin{aligned}
C_{r}(\beta ; \tau)= & a^{2} c_{s, 2,1} D^{-1} e^{-j 2 \pi \beta \varepsilon D} e^{-j \frac{2 \pi}{\rho \kappa} \Delta f_{c} T_{u} \mathrm{\tau}} \\
& * \Gamma_{K}(\tau) \Lambda(\tau), \\
\kappa_{2,1}^{\mathrm{OFDM}}= & \left\{\beta \in[-1 / 2,1 / 2) \mid \beta=l D^{-1}, l\right. \\
= & 1,2,3 \ldots\},
\end{aligned}
$$

where $\quad \Gamma_{K}(\tau)=e^{j \pi(K-1) \tau / \rho K} \sin (\pi \tau) / \sin (\pi \tau / \rho K), \quad \Lambda(\tau)=$ $\sum_{u=-\infty}^{\infty} g(u) g^{*}(u+\tau) e^{-j 2 \pi \beta u}$, and $D=\rho K\left(1+T_{c p} T_{u}{ }^{-1}\right)$ represents the number of samples over an OFDM symbol.

The second-order (one-conjugate) CCs of SCLD and OFDM signals are depicted by CF and delay in Figures 1 and 2, respectively. Note that the normalized form of CF is used in terms of (4) and (6); the sample quantity is taken as the scale of delay.

\section{Blind recognition of OFDM and SCLD signals}

\subsection{Joint time-frequency domain feature function}

At the receiver, suppose that the bandwidth of the signal is roughly estimated, and a low-pass filter is used to remove the out-of-band noise. The signal is downconverted and (over) sampled at a rate of $\rho$ times.

In Section 2, the significant differences between SCLD and OFDM are showed, e.g., in the time domain (when $\mathrm{CF}=0$ ); the CC magnitudes of OFDM signals are nonzero values with delays around $\pm \rho K$, whereas the $C C$ magnitudes of SCLD signals are zero. And in the frequency domain (when delay equals 0 ), $\mathrm{CC}$ magnitudes of SCLD signals appear in peaks with CFs equaling to integer multiples of $1 / \rho$, whereas the $C C$ magnitudes of OFDM are still zero.

Combining the features of OFDM and SCLD in the two domains, we can define a joint time-frequency domain feature function:

$$
z_{J}=\frac{c_{2 r}\left(\rho^{-1} ; 0\right)}{c_{2 r}(0 ; \rho K)}
$$

where $c_{2 r}\left(\alpha, \tau_{n}\right)$ represents the secondary order CCs of the received signal $r(t)$, which is expected to be detected. From Figure 3, we can see that function $z_{J}$ exhibits the obvious difference between OFDM signals and SCLD signals to about 100 times, which is larger than that in a single time domain or a single frequency domain. This reveals that the feature function above may be a better choice in the classification of OFDM and SCLD signals.

Practically, the consistent estimator of the secondorder cyclic cumulants is given as 


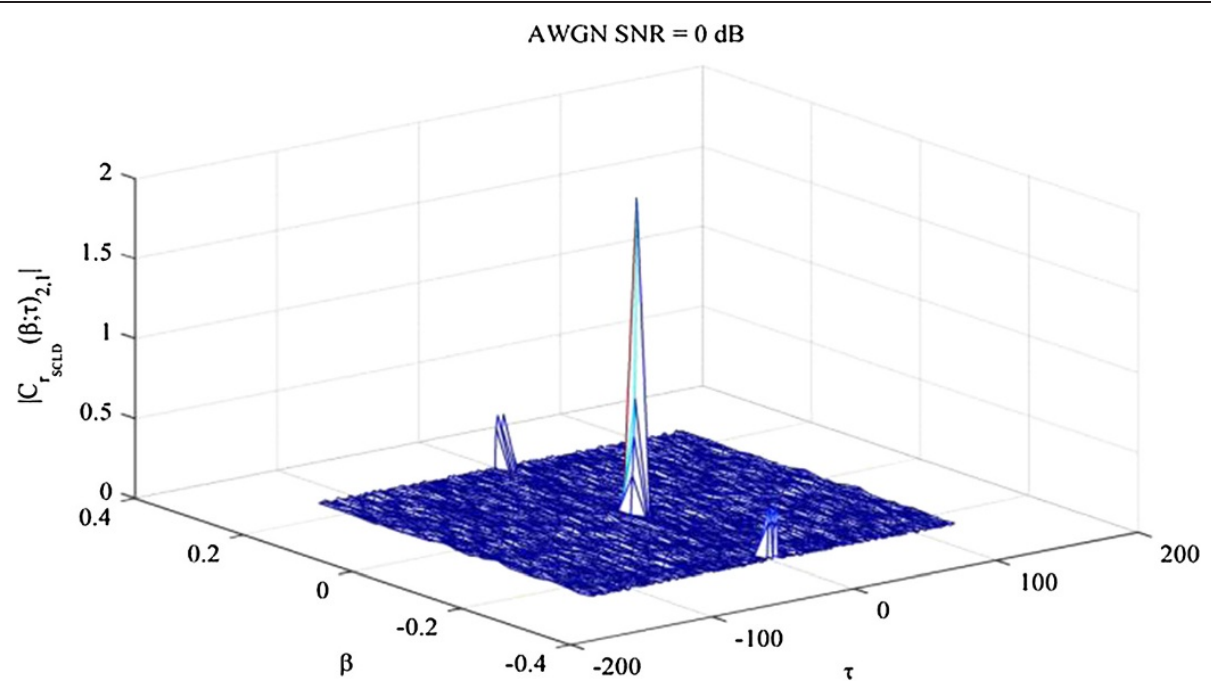

Figure 1 CCs magnitude value of SCLD in AWGN.

$$
\begin{aligned}
\hat{c}_{2 r}(\alpha, \tau) & =\frac{1}{T} \sum_{t=0}^{T-1} r(t) r^{*}(t+\tau) e^{-j \alpha t} \\
& =c_{2 r}(\alpha, \tau)+\varepsilon_{2 r}^{(T)}(\alpha, \tau)
\end{aligned}
$$

where $\varepsilon_{2 r}^{(T)}(\alpha, \tau)$ represents the estimation error that vanishes asymptotically when $T \rightarrow \infty$. Due to the existence of error $\varepsilon_{2 r}^{(T)}(\alpha, \tau)$ the estimator $\hat{c}_{2 r}(\alpha, \tau)$ is seldomly equal to zero practically, even if $\alpha$ is not a cycle frequency.

Plugging (8) into (7), we obtain

$$
z_{J}=\frac{c_{2 r}\left(\rho^{-1} ; 0\right)+\varepsilon_{2 r}^{(T)}\left(\rho^{-1} ; 0\right)}{c_{2 r}(0 ; \rho K)+\varepsilon_{2 r}^{(T)}(0 ; \rho K)} .
$$

Considering an OFDM signal, we can see that the value of $c_{2 r}(0 ; \rho K)$ is larger than that of $\varepsilon_{2 r}^{(T)}(0 ; \rho K)$, and $c_{2 r}\left(\rho^{-1} ; 0\right)$ is zero in noiseless situations. As a result, the decision function of an OFDM signal can be approximately simplified as

$$
z_{\text {J|OFDM }} \approx \frac{\varepsilon_{2 r}^{(T)}\left(\rho^{-1} ; 0\right)}{c_{2 r}(0 ; \rho K)} .
$$

For the SCLD signals, we can get similar conclusions: $c_{2 \mathrm{r}}\left(\rho^{-1} ; 0\right) \gg \varepsilon_{2 \mathrm{r}}^{(T)}\left(\rho^{-1} ; 0\right)$ and $c_{r}(0 ; \rho K)=0$. Therefore, the decision function for SCLD signals can be simplified as

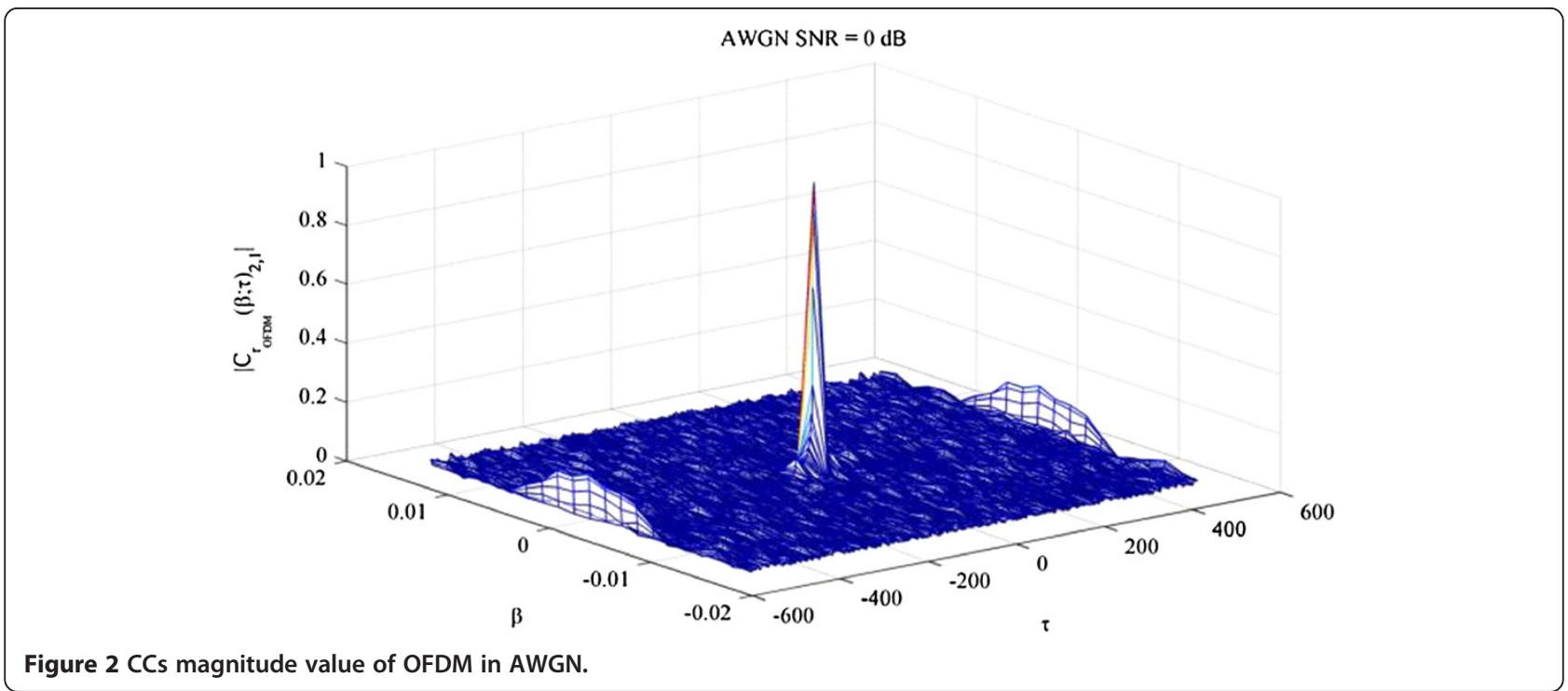




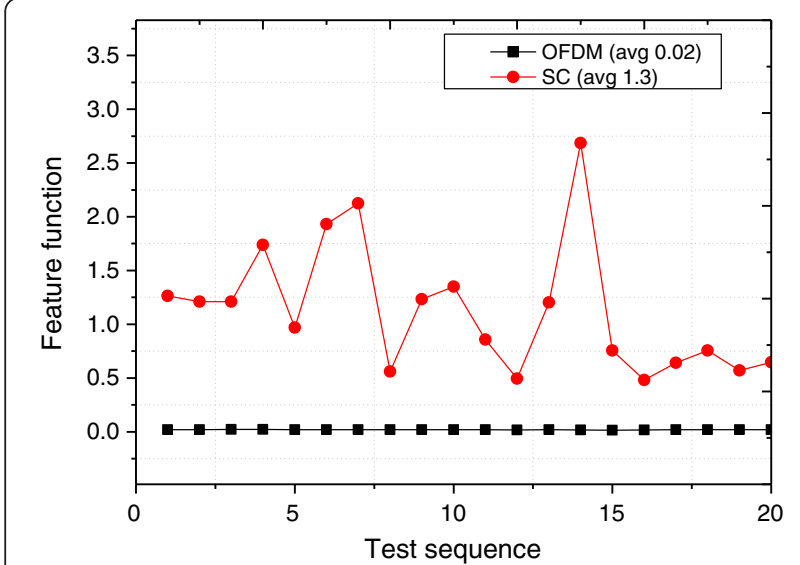

Figure 3 Value range of feature function for OFDM and SC.

$$
z_{J \mid \mathrm{SCLD}} \approx \frac{c_{2 r}\left(\rho^{-1} ; 0\right)}{\varepsilon_{2 r}^{(T)}(0 ; \rho K)}
$$

\subsection{Statistical test framework}

The recognition of OFDM and SCLD signals can be formulated into a hypothesis testing problem:

$$
\begin{aligned}
& \mathrm{H}_{0}: r(t)=\mathrm{S}_{\mathrm{OFDM}}(t)+w(t), \\
& \mathrm{H}_{1}: r(t)=\mathrm{S}_{\mathrm{SCLD}}(t)+w(t) .
\end{aligned}
$$

For simplicity, noise without useful signals is omitted in the statistical test.

This problem is equivalent to a generalized maximumlikelihood detection problem in the cyclic frequency domain. Referring to [10], it defines a generalized likelihood function as the test statistic function:

$$
\gamma_{2 r}(\alpha, \tau)=T \hat{C}_{2 r}^{(T)} \sum_{2 r}{ }^{-1} \hat{C}_{2 r}^{(T)^{\prime}}
$$

where $\hat{C}_{2 r}^{(T)}$ represents a $1 \times 2 \mathrm{~N}$ row vector consisting of second-order $\mathrm{CC}$ estimators:

$$
\begin{aligned}
\hat{C}_{2 r}^{(T)} & =\left[\operatorname{Re}\left\{\hat{c}_{2 r}\left(\alpha, \tau_{1}\right)\right\}, \ldots, \operatorname{Re}\left\{\hat{c}_{2 r}\left(\alpha, \tau_{N}\right)\right\}\right. \\
& \left.=\operatorname{Im}\left\{\hat{c}_{2 r}\left(\alpha, \tau_{1}\right)\right\}, \ldots, \operatorname{Im}\left\{\hat{c}_{2 r}\left(\alpha, \tau_{N}\right)\right\},\right],
\end{aligned}
$$

where $\left\{\tau_{1}, \ldots, \tau_{N}\right\}$ is a fixed set of time delay values, $\operatorname{Re}\{\}$ and $\operatorname{Im}\{\}$ represent the real and imaginary parts. $\sum_{2 r}$ is the asymptotic covariance matrix of $\hat{c}_{2 r}(\alpha, \tau)$. It is also proved that for the frequency $\beta$ which does not belong to CFs, $\gamma_{2 r}(\beta, \tau)$ satisfies chi-squared distribution with 2 $N$ degrees of freedom:

$$
\lim _{T \rightarrow \infty} \gamma_{2 r}(\beta, \tau)=\chi_{2 N}^{2}
$$

Herein, by replacing $\hat{C}_{2 r}^{(T)}$ with $z_{J}$ in (13), we can get the following function:

$$
\gamma_{2 z}(\alpha, \tau)=\frac{\gamma_{2 r}\left(\rho^{-1}, 0\right)}{\gamma_{2 r}(0, \rho K)} .
$$

Based on the analyses in Section 3.1, the testing framework can be transformed into

$$
\begin{aligned}
& \mathrm{H}_{0}: \gamma_{2 z}<\eta \\
& \mathrm{H}_{1}: \gamma_{2 z} \geq \eta,
\end{aligned}
$$

where $\eta$ is the decision threshold.

When $T$ is large enough under the hypothesis of $H_{O}$, we can get $\gamma_{2 r}(0, \rho K) \approx c_{r}(0 ; \rho K)_{2,1} \sum_{2 r}^{-1} c_{r}(0 ; \rho K)_{2,1}$, , which is a constant and is represented by $M_{\mathrm{OFMD}}$. Based on formula (15), we can achieve the statistical distribution of $\gamma_{2 z}$ under $H_{O}$ as follows:

$$
\gamma_{2 z} \mid \mathrm{OFMD}^{\sim} \frac{\chi_{2}^{2}}{M_{\mathrm{OFMD}}} .
$$

Under the hypothesis of $H_{1}$, there is $\gamma_{2 r}\left(\rho^{-1}, 0\right) \approx$ $c_{r}\left(\rho^{-1} ; 0\right)_{2,1} \sum_{2 r}^{-1} c_{r}\left(\rho^{-1} ; 0\right)_{2,1}{ }^{\prime}$, which is also a constant and is represented by $M_{\mathrm{SCLD}}$. The statistical distribution of $\gamma_{2 z}$ under $H_{1}$ is:

$$
\gamma_{2 z} \mid \mathrm{SCLD}^{\sim} \frac{M_{\mathrm{SCLD}}}{\chi_{2}^{2}} .
$$

\subsection{Optimal decision threshold}

In the following, we will analyze the deterministic relation between the probability of correct recognition and the decision threshold value, and then try to derive the optimal threshold value.

In [8], the author proposed an asymptotically optimal $X_{2}$ test in checking of the presence of cyclostationarity. The algorithm used constant false alarm rate to derive the threshold. However, the check of the false rate only took into consideration of the probability that noise is mistakenly recognized as a signal, while neglecting the probability that the existing signal can be mistakenly as noise. Therefore, the derived threshold is not optimal to recognize the signal correctly. In this paper, the proposed statistical test method takes the probability that OFDM is mistaken for SCLD ( $P($ SCLD $\mid$ OFDM $)$ ) into consideration and the probability that SCLD is mistaken for OFDM ( $P($ OFDM $\mid$ SCLD) ) as well. We define the error recognition probability as $P_{\text {error }}=P($ SCLD $\mid$ OFDM $)+$ $P(\mathrm{OFDM} \mid \mathrm{SCLD})$. The rest of this part is to find the optimal threshold and to get the least value of $P_{\text {error }}$ The error recognizing probability can be expressed as

$$
\begin{aligned}
& P(\text { SCLD } \mid \text { OFDM })=P\left(\gamma_{2 z} \geq \eta \mid H_{0}\right) \\
& P(\text { OFDM } \mid \text { SCLD })=P\left(\gamma_{2 z}<\eta \mid H_{1}\right) .
\end{aligned}
$$

Using formulas (18) and (19), we can rewrite formula (20) as 


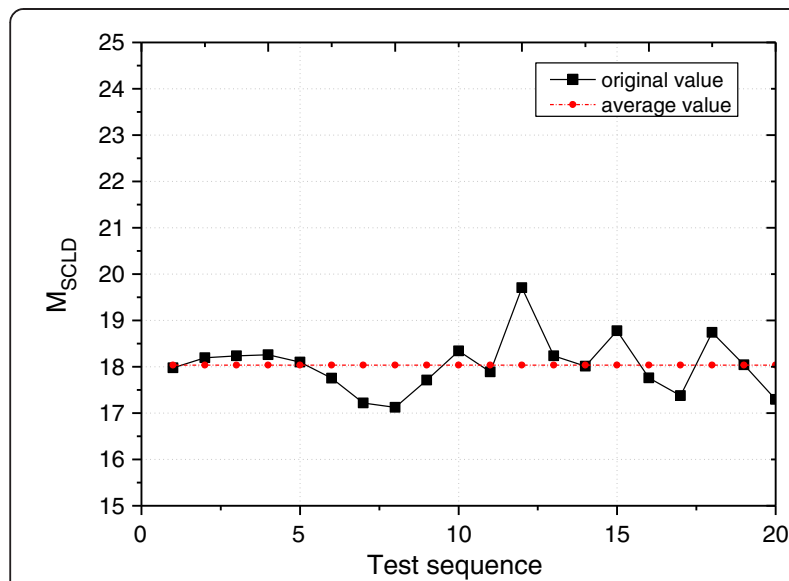

Figure 4 Value of $M_{\mathrm{SCLD}}$.

$$
\begin{aligned}
P_{\text {error }}(\eta) & =P\left(\gamma_{2 z} \geq \eta \mid H_{0}\right)+P\left(\gamma_{2 z}<\eta \mid H_{1}\right) \\
& =e^{\frac{-\eta * M_{\text {OFMD }}}{2}}+e^{\frac{-M_{\text {SCLD }}}{2 \eta}} .
\end{aligned}
$$

In order to find the optimal value $\eta$ of the least error probability $P_{\text {error }}$ we derive Equation 21 and let $P_{\text {error }}^{\prime}(\eta)$ to be zero, then we can get the optimal threshold

$$
\eta=\sqrt{\frac{M_{\mathrm{SCLD}}}{M_{\mathrm{OFMD}}}}
$$

From discussions above, we can conclude that when we do statistic test using the threshold defined by (22), the error recognizing probability is the least. In this condition, the error probabilities for classifying the two kinds of signals are as follows:

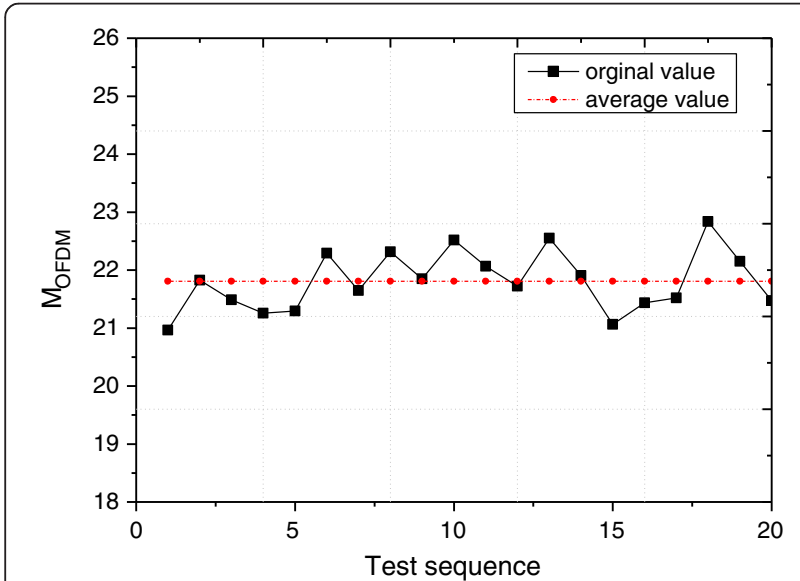

Figure 5 Value of $M_{\text {OFDM }}$.

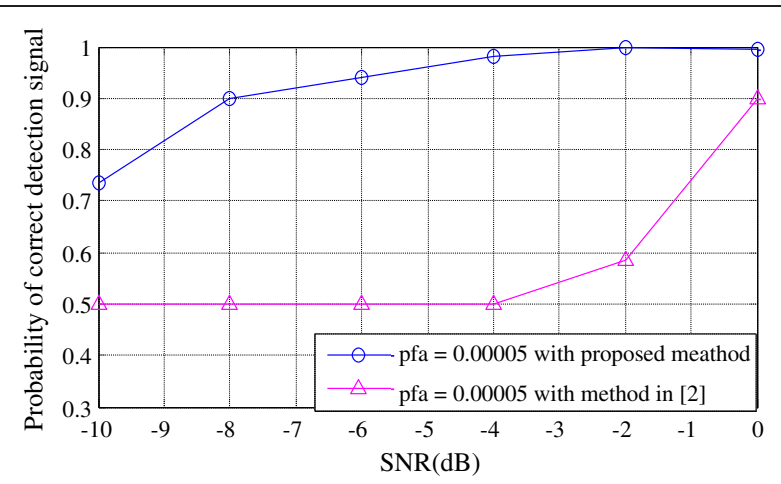

Figure 6 Comparison between the proposed and the original methods in AWGN.

$$
\begin{aligned}
& P(\text { SCLD } \mid \text { OFDM })=P\left(\gamma_{2 z} \geq \eta \mid H_{0}\right) \\
& =P\left(\gamma_{2 r}\left(\rho^{-1}, 0\right)>\sqrt{M_{\mathrm{SCLD}} * M_{\mathrm{OFMD}}}\right), \\
& P(\text { OFDM } \mid \text { SCLD })=P\left(\gamma_{2 z}<\eta \mid H_{1}\right) \\
& =P\left(\gamma_{2 r}(0, \rho K)>\sqrt{M_{\mathrm{SCLD}} * M_{\mathrm{OFMD}}}\right) .
\end{aligned}
$$

\section{Simulation results}

\subsection{Simulation setup}

In the simulation, we adopt QPSK as the modulation mode of the SCLD signals. The transmit filter is a rootraised cosine filter with 0.5 roll-off factor, and the signal bandwidth is $40 \mathrm{kHz}$. For the OFDM signals, QPSK modulation is used on each subcarrier. The signal bandwidth is set to $800 \mathrm{kHz}$, and the number of subcarriers is set to 128 . We set the useful time period of the OFDM symbol to $160 \mu$ s and the cyclic prefix period to $40 \mu \mathrm{s}$. At the transmit side, a raised cosine window with 0.025 roll-off factor is used for OFDM signals. In addition, we set the amplitude factor to 1, timing offset to 0.75 , and frequency offset for SCLD and OFDM signals to 16 and $320 \mathrm{kHz}$. The carrier phases are uniformly distributed over $[-\pi, \pi)$ as a random variable.

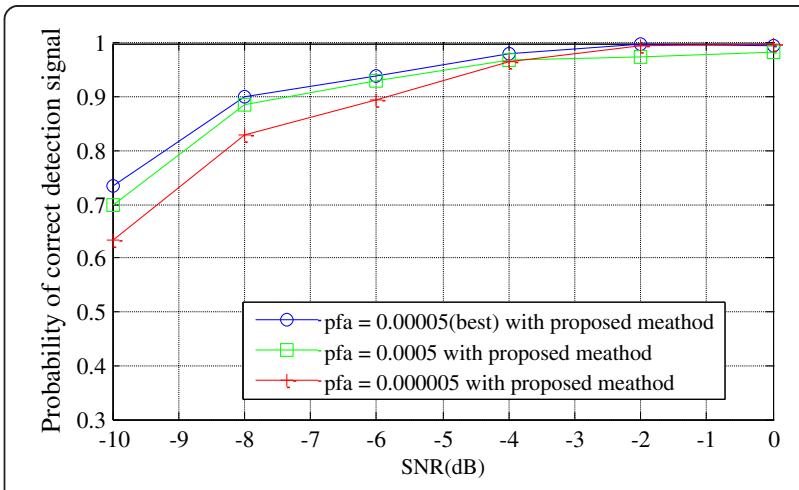

Figure 7 Probability of correct detection signal. 
The received signal is low-pass filtered and (over) sampled, with the factor $\rho$ set to 4 . The final probability of correct recognition equals to the sum of the probabilities when correctly recognizing the OFDM and the SCLD signals. Data for the test is collected from 100 trials of each signal type.

\subsection{Simulation results}

In order to evaluate the performance of the proposed method, we at first compare the proposed method to the method in article [6], in which only the signal features in time domain is used. In the second case, we compare the performance of the proposed method of different $P$ (SCLD $\mid$ OFDM).

Practically, in the simulation, we need to know $M_{\mathrm{SCLD}}$ and $M_{\text {OFDM }}$ previously. From the simulation result in Figures 4 and 5 , we can empirically set $M_{\mathrm{SCLD}} \approx 18$ and $M_{\text {OFDM }} \approx 22$. Base on formula (22), the optimal threshold is $\eta=0.9045$, and $P(\mathrm{SCLD} \mid \mathrm{OFDM}) \approx 5 \times 10^{-5}$, correspondingly.

In the first case, we set the probability that OFDM is mistaken for SCLD to $5 \times 10^{-5}$, so the threshold used in the proposed method is optimal. Figure 6 shows that the proposed method performs better than it in the reference method according to article [6]. As for the reference method, the probability of correct recognition is just about $50 \%$ when SNR varies from -10 to $-4 \mathrm{~dB}$. The reason is that the threshold is too high to detect SCLD, which means that the threshold is irrational, whereas the probability of the proposed method is almost 0.9 at $-8 \mathrm{~dB}$ SNR because the method enlarges the difference between the OFDM and SCLD signals.

In [6], the probability that OFDM is mistaken for SCLD (represented as pfa) is the only error probability considered. In fact, when considering the probability that SCLD may be mistaken for OFDM (represented as pfo), the relationship between the overall error probability and pfa is not linear. In the second case, as is shown in Figure 7, we compare the performance of the proposed method with different pfa values. We can see that when $\mathrm{pfa}=5 \times 10^{-5}$ (corresponding to the optimal threshold), the correct probability is larger than those of when $\mathrm{pfa}=5 \times 10^{-4}$ and $\mathrm{pfa}=5 \times 10^{-6}$. The simulation results verify that the proposed theory and the corresponding method in Section 3 do have good performances for blind modulation recognition.

\section{Conclusions}

The proposed algorithm exploits the nonzero CC features both in time and frequency domains to solve the OFDM and SCLD signal recognition problems. Considering the different features of OFDM and SCLD in two domains, we define a joint feature function to widen the distinguished gap. Based on the defined decision function, the recognition of OFDM and SCLD signals is formulated as a statistical testing problem. The optimal threshold when recognizing the modulation type of received signals is derived to achieve the least error probability. The proposed method in this paper can be extended to recognizing most of the general linear signal modulation types.

\section{Competing interests}

The authors declare that they have no competing interests.

\section{Acknowledgements}

This work was supported by the National Science and Technology Major Project of China under grant 2013ZX03001003-003, and the BUPT Research Innovation Project under grant 2013RC0104.

Received: 24 October 2013 Accepted: 7 January 2014

Published: 16 January 2014

\section{References}

1. D Zeng, X Zeng, H Cheng, B Tang, Automatic modulation classification of radar signals using the Rihaczek distribution and Hough transform. Radar Sonar Navi IET. 6(5), 322-331 (2012)

2. SF Mason, CR Berger, SL Zhou, P Willett, Detection, synchronization, and Doppler scale estimation with multicarrier waveforms in underwater acoustic communication. IEEE J Sel Areas Commun. 26(9), 1638-1649 (2008)

3. WA Gardner, CM Spooner, The cumulant theory of cyclostationary time-series. I. Foundation. Signal Process IEEE Transactions. 42(12), 3387-408 (1994)

4. CM Spooner, WA Gardner, The cumulant theory of cyclostationary timeseries. II. Development and applications. Signal Process IEEE Transactions. 42(12), 3409-29 (1994)

5. OA Dobre, A Abdi, Y Bar-Ness, W Su, Survey of automatic modulation classification techniques: classical approaches and new trends. IET Commun. 1, 261-264 (2007)

6. A Punchihewa, OA Dobre, S Rajan, R Inkol, Cyclostationarity-based algorithm for blind recognition of OFDM and single carrier linear digital modulations (IEEE PIMRC, Athens, Greece, 2007), pp. 3-7

7. A Punchihewa, Q Zhang, OA Dobre, C Spooner, S Rajan, R Inkol, On the cyclostationarity of OFDM and single carrier linearly digitally modulated signals in time dispersive channels: theoretical developments and application. IEEE Trans Wireless Commun. 9(8), 2588-2599 (2010)

8. A Bouzegzi, P Jallon, P Ciblat, A second order statistics based algorithm for blind recognition of OFDM based systems, in IEEE GLOBECOM (, New Orleans, LA, USA, 2008), p. 30

9. A Al-Habashna, OA Dobre, R Venkatesan, DC Popescu, Joint cyclostationarity-based detection and classification of mobile WiMAX and LTE OFDM signals, in IEEE ICC (Kyoto, 2011), pp. 19-22

10. AV Dandawate, GB Giannakis, Statistical test for presence of cyclostationarity. IEEE Trans Signal Process. 42(9), 2355-2369 (1994)

11. M Oner, F Jondral, Cyclostationarity based air interface recognition for software radio systems, in Proceedings of the IEEE Radio and Wireless Conference (, Orlando, FL, 2004), pp. 19-22

12. JG Proakis, Digital Communications, 4th edn. (McGraw Hill, New York, 2000)

13. Z Sun, RZ Liu, WB Wang, Joint time-frequency domain cyclostationaritybased approach to blind estimation of OFDM transmission parameters. EURASIP J Wirel Commun Netw. 2013, 117 (2013)

doi:10.1186/1687-6180-2014-5

Cite this article as: Sun et al:: Cyclostationarity-based joint domain approach to blind recognition of SCLD and OFDM signals. EURASIP Journal on Advances in Signal Processing 2014 2014:5. 